\title{
BMJ Open Households access to improved drinking water sources and toilet facilities in Ethiopia: a multilevel analysis based on 2016 Ethiopian Demographic and Health Survey
}

\author{
Zewudu Andualem (D) , ${ }^{1}$ Henok Dagne, ${ }^{1}$ Zelalem Nigussie Azene, ${ }^{2}$ \\ Asefa Adimasu Taddese (D) , ${ }^{3}$ Baye Dagnew (D) , ${ }^{4}$ Roman Fisseha, \\ Atalay Goshu Muluneh, ${ }^{3}$ Yigizie Yeshaw (i) ${ }^{4}$
}

To cite: Andualem Z, Dagne $\mathrm{H}$, Azene ZN, et al. Households access to improved drinking water sources and toilet facilities in Ethiopia: a multilevel analysis based on 2016 Ethiopian Demographic and Health Survey. BMJ Open 2021;11:e042071. doi:10.1136/ bmjopen-2020-042071

- Prepublication history and additional material for this paper is available online. To view these files, please visit the journal online (http://dx.doi.org/10. 1136/bmjopen-2020-042071)

Received 30 July 2020 Revised 26 February 2021 Accepted 01 March 2021

\section{A) Check for updates}

(C) Author(s) (or their employer(s)) 2021. Re-use permitted under CC BY-NC. No commercial re-use. See rights and permissions. Published by BMJ.

For numbered affiliations see end of article.

\section{Correspondence to} Mr Zewudu Andualem; Zewuduandualem12@gmail. com

\section{ABSTRACT}

Objective This study aimed to assess households access to improved drinking water sources and sanitation facilities and their associated factors in Ethiopia.

Design Cross-sectional study.

Setting Ethiopia.

Participants Household heads.

Primary outcomes Access to improved drinking water sources and toilet facilities.

Methods We conducted an in-depth secondary data analysis of 2016 Ethiopian Demographic and Health Survey. Data from a total of 16650 households and 645 clusters were included in the analysis. The households were selected using a stratified two-stage cluster sampling technique. Multilevel binary logistic regression analyses were performed to identify factors associated with access to an improved drinking water source and toilet facilities. Adjusted OR with a $95 \% \mathrm{Cl}$ was reported with $\mathrm{p}$ value $<0.05$ was used to declare a significant association between the covariates and the outcome variables.

Results The proportions of households' access to improved sources of drinking water and toilet facilities were $69.94 \%$ (95\% Cl: $69.23 \%$ to $70.63 \%$ ) and $25.36 \%$ (95\% Cl: $24.69 \%$ to $26.03 \%$ ), respectively. Households headed by women and households with a better wealth index were positively associated with access to improved drinking water sources. Whereas rural households, $\geq 30$ min round trip to obtain drinking water and region were factors negatively associated with households access to improved drinking water sources. A higher probability of having access to improved toilet facilities: households with heads who had attained higher education, households having better access to improved sources of drinking water and households with better wealth index. While the following households were less likely to have access to improved toilet facilities: households with heads were widowed, households with four to six members, rural households and region

Conclusion The study found that the proportions of households' access to improved drinking water sources and toilet facilities in Ethiopia were relatively low, which
Strengths and limitations of this study

- The use of nationally representative data that can enhance the generalisability of the findings is one of the strengths of this study.

- This study used data from a cross-sectional survey; as a result, the results from analysing the data cannot establish causal relationships.

- Moreover, important variables such as culture, traditions and social norms were not available in Ethiopian Demographic and Health Survey data which could influence the use of toilet facility type.

demands the need to tailor strategies to increase the coverage of access to improved drinking water sources and toilet facilities.

\section{INTRODUCTION}

Access to safe water and basic sanitation is one of the fundamental human rights, and an essential step towards improving living standards to maintain and improve health, human growth and development. ${ }^{1-3}$ It is one of the critical sustainable development challenges. Sustainable Development Goal 6 aims to ensure the availability and sustainable management of water and sanitation for all by $2030 .^{4}$

Unimproved sources of drinking water and sanitation facilities are responsible for increased risks of various infectious diseases such as; cholera, typhoid, schistosomiasis, infections of the respiratory systems, skin and eye. ${ }^{156}$ The currently available evidences also indicate that to prevent the novel coronavirus 
disease outbreak, the provision of safe water, sanitation and hygienic conditions is crucial. ${ }^{7}$

Globally, 2 billion people lack basic sanitation services and 785 million people have no access to clean water. ${ }^{8}$ More than 1.9 million deaths and 123 million disabilityadjusted life-years (DALYs) could have been prevented by the provision of adequate access to water, hygiene and sanitation (WASH) worldwide. The WASH-attributable disease burden accounts for $4.6 \%$ of global DALYs and $3.3 \%$ of global mortality. ${ }^{9}$ About $13 \%$ of under-five mortality was accounted by WASH-attributable disease. ${ }^{10}$ In Ethiopia, $60 \%-80 \%$ of communicable diseases are attributed to limited access to safe water, inadequate sanitation and hygiene services. ${ }^{11}$

Regarding factors associated with improved drinking water sources and toilet facilities: Sex of household heads, region, residence, family size and time to get to drinking water source, age of household head, educational level and marital status of the household heads were predictors linked to access to improved drinking water sources and toilet facilities reported by previous studies. ${ }^{2}{ }^{12-16}$

In sub-Saharan Africa, particularly in Ethiopia, access to improved sources of drinking water and toilet facilities is still lacking and people are practicing open defecation. ${ }^{17} 18$ There is no study in Ethiopia using nationally representative data (2016 Ethiopian Demographic and Health Survey (EDHS) data), which accounts for the hierarchical nature of the data. However, national studies conducted in sub-Saharan African countries in 2014 indicated the spatial variation in the coverage of households' use of improved drinking water supply and sanitation. ${ }^{19}$ Therefore, the current study aimed to assess access to improved drinking water sources and toilet facilities and their associated factors in Ethiopia using EDHS 2016 data.

\section{METHODS}

\section{Patient and public involvement}

This study was based on a publicly available data set (EDHS 2016). Thus, there were no patients or members of the public involved.

\section{Study design and area}

The 2016 main EDHS was a cross-sectional survey conducted from 18 January 2016 to 27 June 2016 in Ethiopia by the Ethiopian Central Statistical Agency. For our case, we used an in-depth secondary data analysis of the survey. It was the fourth survey conducted in each of the 11 regions of Ethiopia (9 regional states and 2 administrative cities). Administratively, each region in Ethiopia is divided into Zones, each Zone, in turn, is divided into Woredas, and each Woreda into Kebeles (the lowest administrative units in the country). ${ }^{20}$

\section{Data sources}

Data were obtained from EDHS 2016 after being registered as an authorised user. The survey collects data on key indicators of health and health-related events including access to improved drinking water sources and toilet facilities. A total of 16650 households from 645 enumeration areas (EAs) were included in the survey. ${ }^{20}$

\section{Sample size and sampling procedure}

A two-stage stratified cluster sampling technique was employed to select study participants. Sampling frame of the 2007 population and housing censuses in which EAs were the sampling units for the first stage and households for the second stage was used. A total of 18008 households were selected for the sample, of which 17067 were occupied. Of the occupied households, 16650 were successfully interviewed, yielding a response rate of $98 \% .{ }^{20}$ The current study included individual-level data for 16650 household heads as well as community characteristics of 645 clusters.

\section{Variables of the study}

Outcome variables

Access to improved drinking water sources and improved toilet facilities.

\section{Explanatory variables}

The explanatory variables for this study were classified as individual-level and community-level factors for both outcome variables. The individual-level factors for the outcome variables were; sex, age, educational level and marital status of the household head. Whereas, the community-level factors for the first outcome variable (access to improved drinking water sources) were; household wealth index, time to get to drinking water sources, family size, place of residence and region. While, for the second outcome variable (access to improved toilet facilities) the community-level factors were; household wealth index, household size, sources of drinking water, place of residence and region. The variables were selected based on the literature review for factors affecting access to improved drinking water sources and improved toilet facilities. The basis of the classification of explanatory variables into the individual-level, household-level and community-level variables were based on previous studies ${ }^{2122}$ and our professional judgements.

\section{Operational definitions}

Improved sources of drinking water:

A household is said to have access to an improved drinking water source if it has water piped into its dwelling, water piped to a yard/plot, a public tap/standpipe, a tube well/ borehole, a protected dug well, a protected spring, rainwater, bottled water or sachet water. ${ }^{23}$

\section{Improved types of toilet facilities:}

A household is said to have access to improved toilet facilities if it has unshared flush/pour flush to piped sewer systems, septic tanks or pit latrines, ventilated improved pit latrines, composting toilets or pit latrines with slabs. ${ }^{23}$ 


\section{Data processing and analysis}

Statistical analysis was performed using Stata V.16 software. The weighted frequencies and the percentages (based on the population size of each region) were computed. The detailed weighting procedure is described elsewhere. ${ }^{20}$

Multilevel binary logistic regression model was used to assess the impact of individual-level and communitylevel factors on households' access to improved sources of drinking water and improved toilet facilities. The model, which is most appropriate to consider the cluster random effect in a multivariate setting and the reason to apply multilevel modelling was the nature of the data collected which have a hierarchical or clustered structure. The first level represents the individual and household and the second level factor is the clusters. Four models were tested in each of the cases (access to improved sources of drinking water and toilet facilities). Model 0 (the null model) was fitted without explanatory variables to test random variability in the intercept and to estimate the intraclass correlation coefficient (ICC). Model I was used to investigate the impact of individual-level factors on the likelihood of having access to improved sources of drinking water and toilet facilities. Model II was used to assess the impact of community-level factors on the likelihood of having access to improved sources of drinking water and toilet facilities. Model III was employed to assess the impact of individual-level and communitylevel factors altogether on access to improved sources of drinking water and toilet facilities.

The random effects (variation of effects) were measured by ICC, percentage change in variance (PCV), median OR (MOR) and deviance (-2 log-likelihood), which measure the variability between clusters in the multilevel models. The ICC explains the cluster variability, while MOR is used to quantify unexplained cluster variability (heterogeneity). The MOR was used to translate cluster variance into OR scale. ${ }^{24-26}$ In the multilevel model, deviance can measure the total variation due to factors at the community and individual levels. ${ }^{25} 27$

Adjusted OR (AOR) with a 95\% CI was reported with $\mathrm{p}$ value $<0.05$ was used to declare a significant association among covariates and outcome variables. A multicollinearity test was performed to rule out if there was a significant correlation between explanatory variables. If the values of variance inflation factor was lower than 10 , then the collinearity problem was considered less likely. ${ }^{28}$

\section{RESULTS}

Socio-demographic characteristics of the study participants

A total of 16650 study participants were included in the study. The median $( \pm \mathrm{SD})$ age of the household heads was 40 years $( \pm 16.22$ years, range $15-95)$. Approximately, 7 out of $10(69.94 \%)$ of the households had access to improved sources of drinking water and only one-fourth $(25.36 \%)$ of households had access to improved sources of toilet facilities. Majority $(98.37 \%)$ of respondents had no formal education. About $68.55 \%$ of households were male-headed (table 1).

Regional distribution in terms of improved drinking water and toilet facility sources in Ethiopia

Among Ethiopian regions, nearly all households in Addis Ababa (99.40\%) had access to improved sources of drinking water. On the contrary, Somali $(56.52 \%)$ and Afar regions (51.39\%) had the least access to improved sources of drinking water. Households in BenishangulGumuz and Amhara regions were lowest in access to toilet facilities with $4.30 \%$ and $6.31 \%$, respectively, while households in Dire Dawa and Addis Ababa had the highest access to improved toilet facilities with $65.20 \%$ and $79.31 \%$, respectively (online supplemental table S1) (table 1).

\section{Factors associated with access to improved drinking water sources}

The proportion of households which had access to improved sources of drinking water was $69.94 \%$ at (95\% CI: $69.23 \%$ to $70.63 \%$ ). Both individual-level and community-level factors had an impact on accessing improved drinking water sources in this study. Among individual-level factors; sex of the household head and from community-level factors; wealth index, time to get to drinking water sources, residence and region were significantly associated with access to improved drinking water sources.

Female-headed households were $1.18 \quad(\mathrm{AOR}=1.18$, 95\% CI: 1.01 to 1.37 ) times more likely to have access to improved drinking water sources than male-headed households.

Compared with poorest households, poorer $(\mathrm{AOR}=1.48$, 95\% CI: 1.26 to 1.74 ), middle-income (AOR $=2.42,95 \%$ CI: 2.03 to 2.90$)$, richer $(\mathrm{AOR}=3.26,95 \%$ CI: 2.68 to 3.97$)$ and richest (AOR=6.97, 95\% CI: 5.17 to 9.41) households were $1.48,2.42,3.26$ and 6.97 times higher probability to have access to improved drinking water sources, respectively.

In contrast to households who got drinking water in $<30 \mathrm{~min}$, households who got drinking water $\geq 30 \mathrm{~min}$ were $35 \%$ less likely to have access to improved drinking water sources $(\mathrm{AOR}=0.65,95 \% \mathrm{CI}$ : 0.58 to 0.73$)$.

The adjusted odds of access to improved drinking water sources in rural households was $94 \%$ less likely compared with urban households ( $\mathrm{AOR}=0.06,95 \% \mathrm{CI}: 0.03$ to 0.11$)$.

The likelihood of access to improved sources of drinking water was $95 \%$ ( $\mathrm{AOR}=0.05,95 \% \mathrm{CI}$ : 0.01 to 0.21 ) in Afar, $94 \%$ (AOR=0.06, 95\% CI: 0.01 to 0.27) in Amhara, 92\% (AOR $=0.08,95 \%$ CI: 0.02 to 0.35$)$ in Oromia, 98\% (AOR=0.02, 95\% CI: 0.01 to 0.10 ) in Somali, 93\% (AOR $=0.07,95 \%$ CI: 0.02 to 0.30$)$ in Southern Nations, Nationalities, and People's Region (SNNPR) and 82\% (AOR $=0.18,95 \%$ CI: 0.04 to 0.89 ) in Harari compared with access to improved water sources in Addis Ababa city (table 2). 
Table 1 Socio-demographic characteristics of the study participants in Ethiopia, Ethiopian Demographic and Health Survey $2016(n=16650)$

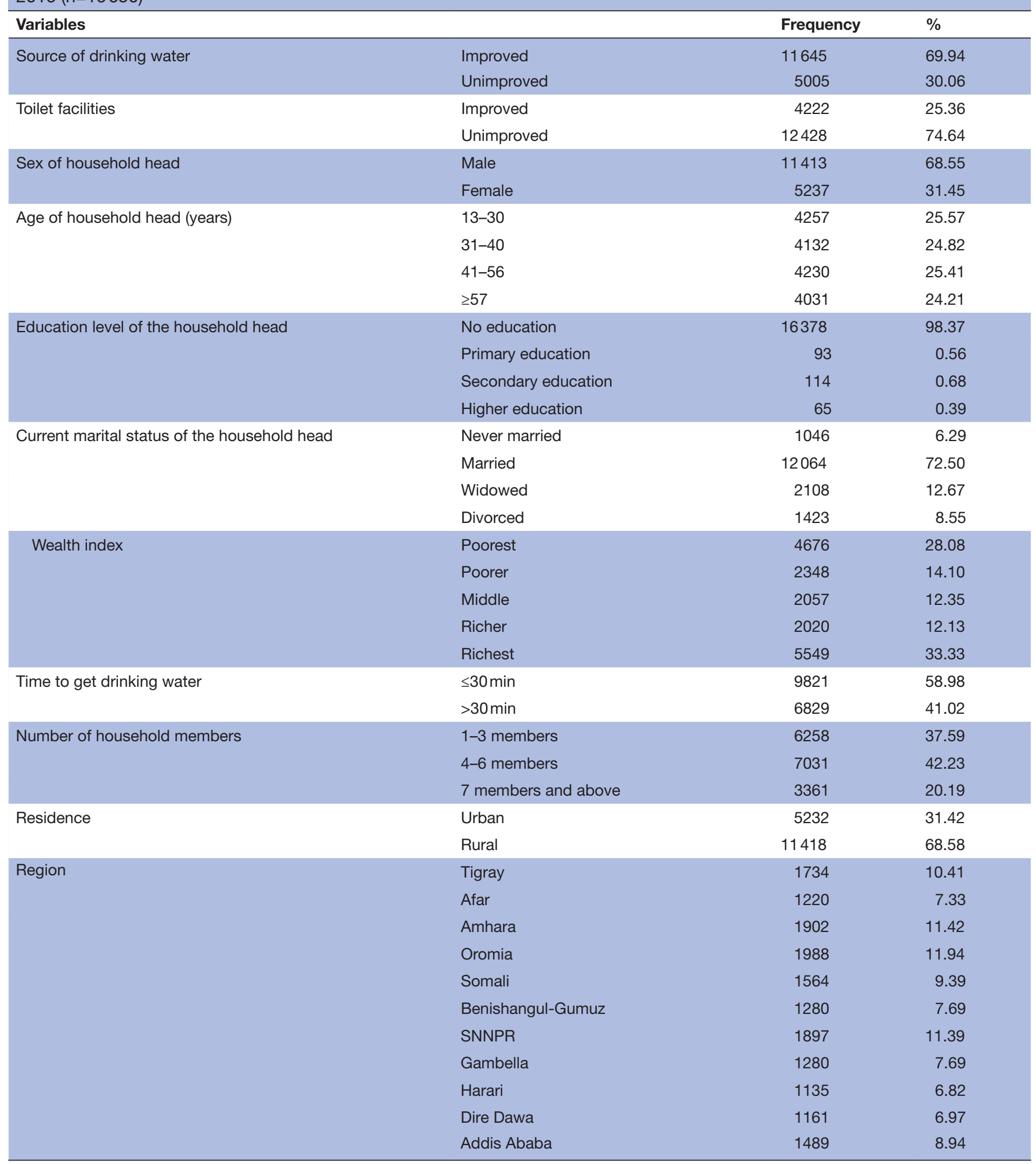

\section{Factors associated with access to improved toilet facilities}

The proportion of households with access to an improved sources of toilet facility was $25.36 \%$ at (95\% CI: $24.69 \%$ to $26.03 \%$ ). Individual-level factors such as educational level and marital status of the household head, and community-level factors such as wealth index, sources of drinking water, number of household members, residence and region were factors significantly impacting households' access to improved toilet facilities.

Compared with household heads that had no formal education, households with heads who attained higher education were 2.21 times more likely to have access to 
Table 2 Multivariable multilevel logistic regression model of the effects of individual-level and community-level factors on improved drinking water sources in Ethiopia, Ethiopian Demographic and Health Survey 2016

\begin{tabular}{|c|c|c|c|c|c|}
\hline \multirow{2}{*}{ Variables } & & \multirow[b]{2}{*}{ Model 0} & \multirow{2}{*}{$\begin{array}{l}\text { Model I } \\
\text { AOR }(95 \% \mathrm{Cl}) \\
\end{array}$} & \multirow{2}{*}{$\begin{array}{l}\text { Model II } \\
\text { AOR }(95 \% \mathrm{Cl})\end{array}$} & \multirow{2}{*}{$\begin{array}{l}\text { Model III } \\
\text { AOR }(95 \% \mathrm{Cl})\end{array}$} \\
\hline & & & & & \\
\hline Sex of household head & Male & & 1 & & 1 \\
\hline \multirow[t]{3}{*}{ Age of household head } & $13-30$ & & 1 & & 1 \\
\hline & $31-40$ & & 0.99 (0.85 to 1.14$)$ & & 1.04 (0.89 to 1.22$)$ \\
\hline & $41-56$ & & 1.07 (0.92 to 1.24$)$ & & $1.13(0.96$ to 1.33$)$ \\
\hline \multirow{4}{*}{$\begin{array}{l}\text { Education level of the } \\
\text { household head }\end{array}$} & No education & & 1 & & 1 \\
\hline & Primary & & 0.93 (0.50 to 1.73 ) & & 0.98 (0.52 to 1.83$)$ \\
\hline & Secondary & & 1.69 (0.82 to 3.49$)$ & & 1.74 (0.83 to 3.65$)$ \\
\hline & Higher & & $1.51(0.40$ to 5.70$)$ & & 1.37 (0.34 to 5.55$)$ \\
\hline $\begin{array}{l}\text { Current marital status of the } \\
\text { household head }\end{array}$ & Never married & & 1 & & 1 \\
\hline \multirow[t]{5}{*}{ Wealth index } & Poorest & & & 1 & 1 \\
\hline & Poorer & & & $1.47(1.25 \text { to } 1.73)^{\star \star}$ & $1.48(1.26 \text { to } 1.74)^{\star \star *}$ \\
\hline & Middle & & & $2.41(2.01 \text { to } 2.88)^{\star \star}$ & $2.42(2.03 \text { to } 2.90)^{* *}$ \\
\hline & Richer & & & $3.23(2.66 \text { to } 3.92)^{\star *}$ & $3.26(2.68 \text { to } 3.97)^{\star \star}$ \\
\hline & Richest & & & $6.84(5.07 \text { to } 9.22)^{\star \star}$ & $6.97(5.17 \text { to } 9.41)^{\star \star}$ \\
\hline \multirow[t]{2}{*}{ Time to get drinking water } & $<30 \mathrm{~min}$ & & & 1 & 1 \\
\hline & $\geq 30$ min & & & $0.66(0.58 \text { to } 0.74)^{\star \star}$ & $0.65(0.58 \text { to } 0.73)^{* \star}$ \\
\hline \multirow{3}{*}{$\begin{array}{l}\text { Number of household } \\
\text { members }\end{array}$} & 1-3 members & & & 1 & 1 \\
\hline & 4-6 members & & & $0.86(0.77 \text { to } 0.97)^{\star}$ & 0.88 (0.77 to 1.00$)$ \\
\hline & $\begin{array}{l}7 \text { members and } \\
\text { above }\end{array}$ & & & 0.88 (0.76 to 1.01$)$ & 0.88 (0.75 to 1.04$)$ \\
\hline \multirow{8}{*}{ Region } & Oromia & & & $0.08(0.02 \text { to } 0.35)^{\star \star}$ & $0.08(0.02 \text { to } 0.35)^{\star *}$ \\
\hline & Somali & & & $0.02(0.01 \text { to } 0.10)^{* *}$ & $0.02(0.01 \text { to } 0.10)^{\star \star}$ \\
\hline & $\begin{array}{l}\text { Benishangul- } \\
\text { Gumuz }\end{array}$ & & & $0.39(0.08$ to 1.80$)$ & 0.39 (0.08 to 1.81$)$ \\
\hline & SNNPR & & & $0.07(0.02 \text { to } 0.30)^{\star *}$ & $0.07(0.02 \text { to } 0.30)^{\star \star}$ \\
\hline & Gambella & & & 0.30 (0.06 to 1.35 ) & 0.29 (0.06 to 1.33$)$ \\
\hline & Harari & & & $0.18(0.04 \text { to } 0.88)^{\star}$ & $0.18(0.04 \text { to } 0.89)^{*}$ \\
\hline & Dire Dawa & & & 0.33 (0.07 to 1.64$)$ & 0.33 (0.07 to 1.63$)$ \\
\hline & Addis Ababa & & & 1 & 1 \\
\hline \multirow[t]{4}{*}{ Random effect } & $\begin{array}{l}\text { Community level } \\
\text { variance (SE) }\end{array}$ & $12.24(1.13)^{\star \star}$ & $12.05(1.11)^{\star \star}$ & $4.75(0.42)^{\star \star}$ & $4.73(0.41)^{\star \star}$ \\
\hline & ICC (\%) & 78.81 & 78.55 & 59.08 & 58.97 \\
\hline & MOR & 9.05 & 8.96 & 5.64 & 5.62 \\
\hline & PCV & Reference & $1.55 \%$ & 61.19 & $61.36 \%$ \\
\hline \multirow[t]{2}{*}{ Model fit statistics } & Log-likelihood & -5997.01 & -5987.89 & -5619.38 & -5611.48 \\
\hline & Deviance & 11994.02 & 11975.78 & 11238.76 & 11222.96 \\
\hline
\end{tabular}

Significant at $\mathrm{p}<0.05 ;{ }^{* *} \mathrm{p}<0.001$

AOR, adjusted OR; ICC, intraclass correlation coefficient; Model 0, empty (null) model; Model I, only individual-level explanatory variables included in the model; Model II, only community-level explanatory variables included in the model; Model III, combined model; both individual-level and community-level explanatory variables; MOR, median OR; PCV, proportional change in variance. 
improved toilet facilities at $(\mathrm{AOR}=2.21,95 \% \mathrm{CI}: 1.12$ to 4.36). The likelihood of access to improved toilet facilities was $25 \%$ (AOR $=0.75,95 \%$ CI: 0.57 to 0.98$)$ lower in households with heads who were widowed compared with those who never married.

Compared with poorest households' access to improved toilet facilities, households with the following wealth indices have the following positive associations: poor households had a (AOR=3.97, 95\% CI: 2.99 to 5.29) higher probability, middle households had a $(\mathrm{AOR}=5.82,95 \%$ CI: 4.35 to 7.80 ) higher probability, richer households had a (AOR=8.58,95\% CI: 6.40 to 11.50$)$ higher probability and richest households had a $(\mathrm{AOR}=23.94,95 \% \mathrm{CI}$ : 17.45 to 32.83 ) higher probability.

Households who had improved sources of drinking water were 1.37 (AOR=1.37,95\% CI: 1.12 to 1.66 ) times more likely to have access to improved toilet facilities compared with their counterparts.

Households with more members were less likely to have access to improved toilet facilities. Compared with households with one to three members, households with four to six members had a $24 \%$ (AOR=0.86, $95 \%$ CI: 0.75 to $0.98)$ lower probability to have access to improved toilet facilities.

In this study, a decrease in the probability of access to improved toilet facilities was observed for rural households contrary to urban households by $72 \% \quad(\mathrm{AOR}=0.28$, 95\% CI: 0.20 to 0.38 ).

About $72 \%$ in Tigray (AOR=0.28, 95\% CI: 0.17 to 0.46 ), $63 \%$ in Afar (AOR=0.37, 95\% CI: 0.21 to 0.67 ), 96\% in Amhara (AOR $=0.04,95 \%$ CI: 0.02 to 0.06 ), $90 \%$ in Oromia (AOR $=0.10,95 \%$ CI: 0.06 to 0.17 ), $95 \%$ in Benishangul-Gumuz (AOR $=0.05,95 \%$ CI: 0.03 to 0.10 ), $80 \%$ in SNNPR (AOR $=0.20,95 \%$ CI: 0.12 to 0.33 ), $85 \%$ in Gambella (AOR $=0.15,95 \%$ CI: 0.09 to 0.26 ) and $60 \%$ in Harari (AOR $=0.40,95 \%$ CI: 0.24 to 0.66 ) households had a higher probability to have access to improved toilet facilities as compared with households in Addis Ababa city (table 3 ).

\section{Random-effects analysis}

The prevalence of access to improved drinking water sources varied across communities (community-level variance $=12.24, p \leq 0.001$ ) (table 2 ). The null model (Model 0) revealed that $78.81 \%$ of the total variance in the odds of access to improved drinking water sources was accounted by between-cluster variation of characteristics $(\mathrm{ICC}=0.7881)$. The inter-cluster variability declined over successive models, from $78.81 \%$ in the null model to $78.55 \%$ in the only individual-level, $59.08 \%$ in the only community-level and $58.97 \%$ in the final (combined) models. The proportional change in variance indicated that the addition of predictors to the empty model explained an increased proportion of variation in access to improved drinking water sources. The combined model showed that a higher PCV, that is, $61.36 \%$ of the variance in access to improved drinking water sources could be explained by the combined factors at the individual-level and community-level factors.

In the final model (Model III) (table 3), ICC value of 0.2708 displayed that $27.08 \%$ of the total variation in access to improved toilet facilities is accounted for the community-level factors. The remaining $72.92 \%$ variation is therefore triggered by the individual-level and other unknown factors. The proportional change in variance indicated that the addition of predictors to the empty model explained an increased proportion of variation in access to improved toilet facilities. The combined model showed a higher PCV, that is, $85.91 \%$ of the variance in access to improved toilet facilities could be explained by the combined factors at the individual-level and community-level factors.

\section{Model fit statistics}

As shown in tables 2 and 3 (model fit statistics), the values of log-likelihood and deviance showed a subsequent reduction which demonstrated that each model represents a significant improvement over the previous model and it points to the goodness of fit for the final model built in the analysis.

\section{DISCUSSION}

In this study, we aimed to assess access to improved drinking water sources and toilet facilities and their associated factors in Ethiopia. Accordingly, the proportion of households' access to improved drinking water sources was $69.94 \%$ (95\% CI: $69.23 \%$ to $70.63 \%$ ). This finding is lower than reports from studies conducted in Ghana, ${ }^{2}$ Viet $\mathrm{Nam}^{16}$ and Eswatini. ${ }^{14}$ Whereas, it is higher than a reported proportion from a study conducted in Nepal. ${ }^{15}$ The proportion of households' access to toilet facilities was $25.36 \%$ (95\% CI: $24.69 \%$ to $26.03 \%$ ). This result is higher than the one reported from Ghana, ${ }^{2}$ however, lower than studies from Nigeria ${ }^{29}$ and Viet Nam. ${ }^{16}$ The variation could be the disparities in gross domestic product (GDP) status and literacy rate among countries, study period and setting.

Individual-level factors were associated with both access to improved drinking water sources and toilet facilities in the present study. Female-headed households were 1.18 times more likely than male-headed households to access improved drinking water sources. Similar findings were reported from Ghana, Viet Nam and Nigeria. ${ }^{2}{ }^{13} 16$ Gender differences may play an important role in the work division in developing countries. Most of the time women have higher household responsibilities such as fetching water, cleaning compounds, childcare and food preparation. Therefore, this might be directly linked with water and sanitation in the sense that women may be preoccupied with other daily routines than WASH.

Those households with heads having better educational status were 2.21 times more likely to access improved toilet facilities. This study finding is supported by evidences from previous studies. ${ }^{142930}$ Households who are led by 
Table 3 Multivariable multilevel logistic regression model of the effects of individual-level and community-level factors on improved sources of toilet facilities in Ethiopia, Ethiopian Demographic and Health Survey 2016

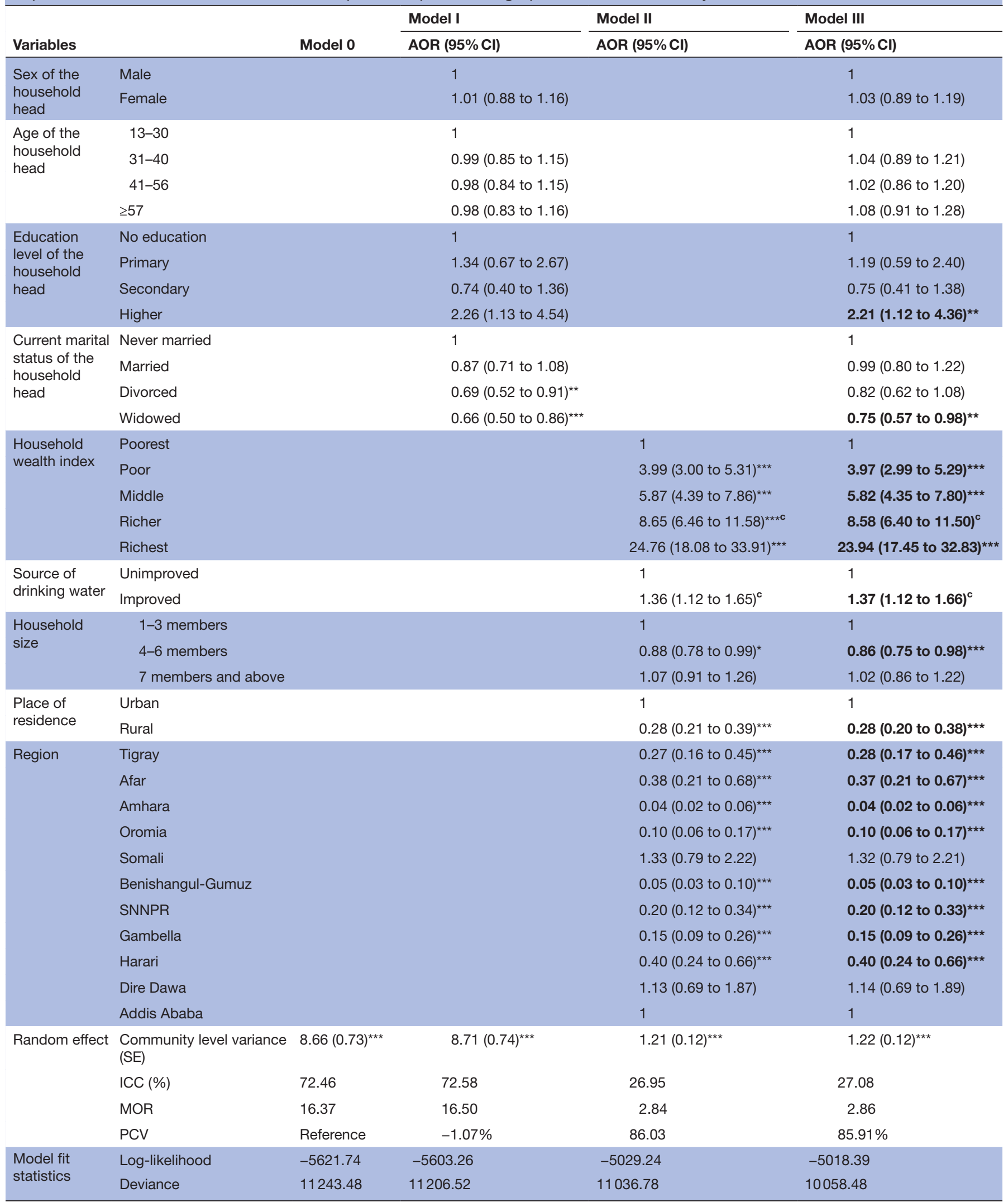

Significant at ${ }^{*} \mathrm{p}<0.05 ;{ }^{* *} \mathrm{p}<0.01 ;{ }^{* * *} \mathrm{p}<0.001$.

AOR, adjusted OR; ICC, intraclass correlation coefficient; Model 0, empty (null) model; Model I, only individual-level explanatory variables included in the model; Model II, only community-level explanatory variables included in the model; Model III, combined model; both individual-level and community-level explanatory variables; MOR, median OR; PCV, proportional change in variance. 
heads who have no education had a lower probability of access to improved toilet facilities. In sub-Saharan Africa, education is a resource factor of quality health outcomes, and educated people usually are more aware of the condition that guarantees their well-beings. This implies that educated household heads in this study may have used their resources to provide their households with improved toilet facilities.

In addition to individual-level factors, community-level factors were also a significant factor in determining access to improved drinking water sources and toilet facilities. Compared with households within the poorest category, households within the poor, middle, richer and richest wealth index category were 1.48, 2.42, 3.26 and 6.97 times more likely to have access to improved sources of drinking water and 3.97, 5.82, 8.58 and 23.94 times higher odds to access to improved toilet facilities, respectively. This result is consistent with results from previous studies. ${ }^{13} 1431$ People who have better incomes would intend to fulfil the necessities of life. Economically, the rich can afford the initial high cost of both water and sanitation facilities and the poor may be disproportionately underserved in the distribution of public utility, and hence consume poor quality water and use unimproved toilet facilities.

Compared with households that got drinking water $<30 \mathrm{~min}$, households which obtained drinking water $\geq 30$ min were $35 \%$ less likely to access improved drinking water sources. Hence, the length of time to get drinking (proximity of a house to a drinking water source) water might make difference in access to improved sources of drinking water and coverage (\%) with improved water supply (Household connection, public standpipes, protected underground water sources, rainwater collection). This is not surprising as physical distance is one of the reasons of WASH service inaccessibility.

The adjusted odds of access to improved drinking water sources and toilet facilities were $94 \%$ and $72 \%$ less likely among rural households as compared with urban households, respectively. The rural-urban disparity in access to improved sources of drinking water and toilet facilities have been reported by several other previous studies, too. ${ }^{12} 3233$ The disparity might be, in sub-Saharan Africa, most of the people live in rural areas and their economic status is poor. Therefore, they do not have adequate financial resources to acquire improved drinking water sources and toilet facilities.

Furthermore, households who had improved sources of drinking water were 1.37 times more likely to access improved toilet facilities. This study finding is consistent with an earlier study. ${ }^{29}$ The possible explanation could be a lack of access to adequate sanitation is also linked to the limited access to water supply and households who had improved water sources may be practicing more in hygiene and sanitation.

Lastly, those households with four to six members were $24 \%$ less likely to have access to improved toilet facilities compared with households with one to three members. This is contrary to that of an earlier study. ${ }^{2}$ The possible explanation could be the highest number of household members, the fewer resources they could have to build improved toilet facilities.

The results of our study should be interpreted with the following limitations. Since the information is recorded retrospectively, it might be prone to recall bias, and the analyses were conducted using data collected in a crosssectional survey, which prevents causal inferences.

\section{CONCLUSIONS}

The proportion of households' access to an improved sources of drinking water and toilet facilities was low in Ethiopia. Sex of the household head, household wealth index, time to get drinking water, place of household/ urban/rural residence and region were factors impacting access to improved drinking water sources. Educational level of the household head, household wealth index, marital status of the household head (widowed), sources of drinking water, number of household members, household or rural/urban residence and region were factors associated with access to improved toilet facilities. Thus, we authors recommend governmental and nongovernmental organisations working on WASH should consider a multifaceted policy approach that accounts for the regions and residence variations and other identified factors to ease up the problem.

\section{Author affiliations}

${ }^{1}$ Department of Environmental and Occupational Health and Safety, College of Medicine and Health Sciences, University of Gondar, P. O. Box 196, Gondar, Ethiopia 2Department of Women's and Family Health, School of Midwifery, College of Medicine and Health Sciences, University of Gondar, P. O. Box 196, Gondar, Ethiopia ${ }^{3}$ Department of Epidemiology and Biostatistics, Institute of Public Health, College of Medicine and Health Sciences, University of Gondar, P. 0. Box 196 Gondar, Ethiopia ${ }^{4}$ Department of Human Physiology, School of Medicine, College of Medicine and Health Sciences, University of Gondar, P. 0. Box 196 Gondar, Ethiopia ${ }^{5}$ Department of Medical Microbiology, School of Biomedical and Laboratory Sciences, College of Medicine and Health Sciences, University of Gondar, Gondar, Ethiopia

Acknowledgements We would like to thank the Demographic and Health Survey (DHS) programme for allowing the use of Ethiopian Demographic and Health Survey (EDHS) data for this study.

Contributors ZA wrote the analysed data, drafted the manuscript and prepared the manuscript. HD, ZNA, AAT, BD, RF, AGM and YY involved in drafting, writing - review and editing and commenting the manuscript. All authors read and approved the final manuscript.

Funding The authors have not declared a specific grant for this research from any funding agency in the public, commercial or not-for-profit sectors.

\section{Competing interests None declared.}

Patient consent for publication Not required.

Ethics approval The Ethiopian Demographic and Health Survey 2016 was approved by the National Research Ethics Review Committee of Ethiopia and ICF Macro International. The approval letter was obtained from the measureDemographic and Health Survey (DHS) for the use of this data, and the data set was downloaded from www.meauredhs.com. This analysis was approved by the Department of Environmental and Occupational Health and Safety, University of Gondar.

Provenance and peer review Not commissioned; externally peer reviewed.

Data availability statement Data are available upon reasonable request. Data are available upon reasonable request. 
Supplemental material This content has been supplied by the author(s). It has not been vetted by BMJ Publishing Group Limited (BMJ) and may not have been peer-reviewed. Any opinions or recommendations discussed are solely those of the author(s) and are not endorsed by BMJ. BMJ disclaims all liability and responsibility arising from any reliance placed on the content. Where the content includes any translated material, BMJ does not warrant the accuracy and reliability of the translations (including but not limited to local regulations, clinical guidelines, terminology, drug names and drug dosages), and is not responsible for any error and/or omissions arising from translation and adaptation or otherwise.

Open access This is an open access article distributed in accordance with the Creative Commons Attribution Non Commercial (CC BY-NC 4.0) license, which permits others to distribute, remix, adapt, build upon this work non-commercially, and license their derivative works on different terms, provided the original work is properly cited, appropriate credit is given, any changes made indicated, and the use is non-commercial. See: http://creativecommons.org/licenses/by-nc/4.0/.

\section{ORCID iDs}

Zewudu Andualem http://orcid.org/0000-0002-4731-1696

Asefa Adimasu Taddese http://orcid.org/0000-0001-5506-8705

Baye Dagnew http://orcid.org/0000-0002-2397-5930

Yigizie Yeshaw http://orcid.org/0000-0003-4587-7925

\section{REFERENCES}

1 Prüss-Ustün A, Wolf J, Bartram J, et al. Burden of disease from inadequate water, sanitation and hygiene for selected adverse health outcomes: an updated analysis with a focus on low- and middleincome countries. Int J Hyg Environ Health 2019;222:765-77.

2 Agbadi P, Darkwah E, Kenney PL. A multilevel analysis of Regressors of access to improved drinking water and sanitation facilities in Ghana. J Environ Public Health 2019;2019:1-11.

3 Assembly UG. Resolution on human right to water and sanitation. New York: United Nations, 2010.

4 Brookes JD, Carey CC. Ensure availability and sustainable management of water and sanitation for all. UN Chron 2015;51:15-16.

5 Mills JE, Cumming $\mathrm{O}$. The impact of water, sanitation and hygiene on key health and social outcomes, 2016. Available: https://www Ishtm ac uk/sites/default/files/2017-07/WASHEvidencePaper_HighRes 01

6 Saxena SK, Kumar S, Haikerwal A. Introductory chapter: neglected tropical waterborne infectious diseases-strategies for mitigation. Water Challenges of an Urbanizing World 2018;1.

7 Organization WH. Water, sanitation, hygiene, and waste management for the COVID-19 virus: interim guidance, 23 April 2020. World Health Organization, 2020.

8 Organization WH. Progress on drinking water, sanitation and hygiene: 2017 update and SDG baselines, 2017.

9 Organization WH. Water, sanitation, hygiene and health: a primer for health professionals. World Health Organization, 2019.

10 Organization WH. Safer water, better health, 2019

11 UNICEF. Water, sanitation and hygiene (wash) 2020. [cited 2020 April, 14]. Available: https://www.unicef.org/ethiopia/water-sanitation-andhygiene-wash

12 Mulenga JN, Bwalya BB, Kaliba-Chishimba K. Determinants and inequalities in access to improved water sources and sanitation among the Zambian households. International Journal of Development and Sustainability 2017;6:746-62.

13 Morakinyo OM, Adebowale SA, Oloruntoba EO. Wealth status and sex differential of household head: implication for source of drinking water in Nigeria. Arch Public Health 2015;73:58.

14 Simelane MS, Shongwe MC, Vermaak K. Determinants of Households' Access to Improved Drinking Water Sources: A
Secondary Analysis of Eswatini 2010 and 2014 Multiple Indicator Cluster Surveys. Advances in Public Health, 2020.

15 Wang C, Pan J, Yaya S, et al. Geographic inequalities in accessing improved water and sanitation facilities in Nepal. Int J Environ Res Public Health 2019;16:1269.

16 Tuyet-Hanh TT, Long TK, Van Minh H, et al. Longitudinal household trends in access to improved water sources and sanitation in chi Linh town, HAl Duong Province, Viet Nam and associated factors. AIMS Public Health 2016;3:880-90.

17 Organization WH, UNICEF. Progress on drinking water and sanitation: special focus on sanitation. progress on drinking water and sanitation: special focus on sanitation, 2008.

18 Beyene A, Hailu T, Faris K, et al. Current state and trends of access to sanitation in Ethiopia and the need to revise indicators to monitor progress in the post-2015 era. BMC Public Health 2015; $15: 451$

19 Pullan RL, Freeman MC, Gething PW, et al. Geographical inequalities in use of improved drinking water supply and sanitation across sub-Saharan Africa: mapping and spatial analysis of cross-sectional survey data. PLoS Med 2014;11:e1001626.

20 Central Statistical Agency (CSA). Central Statistical Agency (CSA) [Ethiopia] and ICF Ethiopia demographic and health Survey 2016. Addis Ababa, Ethiopia, and Rockville, Maryland, USA: CSA and ICF, 2017.

21 Simelane M, Shongwe M, Vermaak K, et al. Determinants of Households' Access to Improved Drinking Water Sources: A Secondary Analysis of Eswatini 2010 and 2014 Multiple Indicator Cluster Surveys. 2020, 2020.

22 Ribeiro Sarmento MPH D. An analysis of access to improved drinking water and sanitation and distance to the water source in a newly independent country, Timor-Leste: assessing geographical and socioeconomic disparities, 2015.

23 Supply W, Programme SM, Organization WH, et al. Water for life: making it happen. World health organization, 2005.

24 Merlo J, Chaix B, Ohlsson H, et al. A brief conceptual tutorial of multilevel analysis in social epidemiology: using measures of clustering in multilevel logistic regression to investigate contextual phenomena. J Epidemiol Community Health 2006;60:290-7.

25 Merlo J, Yang M, Chaix B, et al. A brief conceptual tutorial on multilevel analysis in social epidemiology: investigating contextual phenomena in different groups of people. J Epidemiol Community Health 2005;59:729-36.

26 Larsen K, Merlo J. Appropriate assessment of neighborhood effects on individual health: integrating random and fixed effects in multilevel logistic regression. Am J Epidemiol 2005;161:81-8.

27 Spiegelhalter DJ, Best NG, Carlin BP, et al. Bayesian measures of model complexity and fit. J Royal Statistical Soc B 2002;64:583-639.

28 Hair JF, William J, Black Barry J, et al. Multivariate Data Analysis. Edition S, 2018.

29 Abubakar IR. Access to sanitation facilities among Nigerian households: determinants and sustainability implications. Sustainability 2017:9:547.

30 Akpakli DE, Manyeh AK, Akpakli JK, et al. Determinants of access to improved sanitation facilities in rural districts of southern Ghana: evidence from Dodowa health and demographic surveillance site. BMC Res Notes 2018;11:473.

31 Njuguna J. Progress in sanitation among poor households in Kenya: evidence from demographic and health surveys. BMC Public Health 2019;19:135.

32 Irianti S, Prasetyoputra P, Sasimartoyo TP, et al. Determinants of household drinking-water source in Indonesia: an analysis of the 2007 Indonesian family life survey. Cogent Med 2016;3:1151143.

33 Prasetyoputra P, Irianti S. Access to improved sanitation facilities in Indonesia: an econometric analysis of geographical and socioeconomic disparities. Journal of Applied Sciences in Environmental Sanitation 2013;8. 